\title{
Percepción de los hombres y sus parejas sobre la calidad de vida en el climaterio masculino
}

\author{
Alide Salazar M. $P h D^{a}$, Tatiana Paravic K. $P h D^{b}$, Omar A. Barriga PhD. ${ }^{c}$ \\ a Enfermera Matrona, Departamento de Enfermería, Facultad de Medicina, Universidad de Concepción, Becaria CO- \\ NICYT. b Enfermera, Departamento de Enfermería, Facultad de Medicina, Universidad de Concepción. ${ }^{\mathrm{C}}$ Sociólogo, \\ Departamento de Sociología y Antropología, Facultad de Ciencias Sociales, Universidad de Concepción, Chile.
}

\section{RESUMEN}

Objetivo: Determinar si existen diferencias entre la percepción de los hombres y de sus parejas sobre la calidad de vida relacionada con salud en la etapa de climaterio masculino. Método: Diseño descriptivo y correlacional. Población del estudio integrada por 49 parejas, formadas por hombres entre 40-65 años y sus parejas mujeres entre 40-60 años de un Centro de Salud de Concepción, Chile. Las parejas fueron entrevistadas en sus hogares previo consentimiento informado. Ambos respondieron de forma independiente la escala Aging Males' Symptoms. Se utilizó estadística descriptiva, Indice de Kappa y t de Student. Resultados: La edad promedio fue 53,2 años para los hombres y 50,7 años para las mujeres. El $54,8 \%$ de los hombres mostró alguna alteración de la calidad de vida, mostrando mayor alteración en las sub-escalas somatovegetativa y sexual. Al comparar los puntajes de los hombres en las tres sub-escalas, con la percepción de sus parejas, hubo diferencias significativas sólo en la sub-escala psicológica $(p<0,01)$. Conclusión: No existen diferencias significativas en la percepción que los hombres y sus parejas tienen respecto de calidad de vida relacionada con salud de los hombres durante esta etapa, en los aspectos somatovegetativo y sexual, sólo existe diferencia en el aspecto psicológico.

\section{PALABRAS CLAVE: Calidad de vida, climaterio masculino, síntomas de envejecimiento masculino, parejas}

\section{SUMMARY}

Objective: To determine if there are differences between men's and their couples' perception of health related quality of life during the male climacteric period. Method: Cross-sectional and correlational design. The studied sample consisted of 49 couples, made up of men in a range of 40 to 65 years of age and women in a range of 40 to 60 years of age, enrolled in a Community Health Center in Concepción, Chile. Couples were interviewed in their homes after providing informed consent. Both members answered the Aging Males' Symptoms independently. Descriptive statistics, Kappa and Student-t tests were used. Results: The average age was 53.2 years and 50.7 for men and women, respectively. $58.4 \%$ of the men showed any kind of alteration of their quality of life, having more deterioration in the somato-vegetative and sexual subscale. By comparing men scores in three sub-scales to their couples' perception, only psychological sub-scale $(p<0.01)$ presented a significant difference. Conclusion: Men's perception of quality of life related to health during the male climacteric period is not different from their couples' in somato-vegetative and sexual subscales. The psychological subscale is the only exception

KEY WORDS: Quality of life, male climacteric, aging males'symptoms, couples 


\section{INTRODUCCIÓN}

La investigación relativa a la calidad de vida en el climaterio durante las tres últimas décadas ha estado centrada en la mujer, lo que de alguna manera le ha significado un estereotipo. Sin embargo, desde antiguo se ha asociado envejecimiento y declinación de la función testicular, homologándose a lo que ocurre en el sexo femenino con la función ovárica (1), aunque con algunas distinciones. Es así como en la década de 1940 Werner (2) denominó como climaterio masculino al hipogonadismo de inicio tardío que se ha asociado al envejecimiento en hombres sanos. En adelante, la búsqueda de un término apropiado para denominar a esta entidad ha sido objeto de múltiples debates, por lo que se le puede encontrar en la literatura con las siguientes denominaciones: "ADAM" (Androgen Decline of Aging Male), "PADAM" (Partial Androgen Decline of Aging Male), "PEDAM" (Partial Endocrine Deficiency of the Aging Male) y "LOH" (Late Onset Hypogonadism) (3). También se ha utilizado ampliamente el término "andropausia", para describir los cambios físicos y emocionales que se presentan secundariamente en el hombre de edad avanzada asociado a las alteraciones hormonales, sin embargo hay autores que no recomiendan su uso ya que los hombres no experimentan la cesación total de la producción de testosterona ni la pérdida de la capacidad reproductiva (4). Otras denominaciones presentaron Heinemann y cols (5), al publicar la Aging Males' Symptoms rating scale, estos autores utilizan los términos: síntomas de "envejecimiento de los hombres" para los síntomas que ocurren en el rango de edad entre los 40 y los 60 años, y el término "climaterio masculino" si los síntomas/quejas son indicativos de deficiencia hormonal.

A pesar de lo señalado, no ha sido desde fines de la década pasada que investigadores se han preocupado de estudiar el impacto que las manifestaciones del climaterio masculino tienen en la calidad de vida relacionada con salud de los hombres. Es así como su estudio se ha desarrollado de manera insuficiente y casi en su mayoría en países desarrollados. Algunos trabajos utilizando la escala Aging Males' Symptoms han reportando un deterioro variable, particularmente en el ámbito sexual (5-8).

Sin embargo, la investigación en la región de las Américas y en el contexto local ha estado retrasada, siendo unos pocos autores los que se han preocupado por abordarla $(9,10)$. En adición a lo anterior, escasa ha sido el interés por el estudio de este fenómeno en un contexto de parejas, por lo que este estudio tal vez sería el primero en abordar este tema.
El objetivo de esta investigación fue determinar si existen diferencias entre la percepción de los hombres y sus parejas sobre la calidad de vida relacionada con salud en el climaterio masculino.

\section{PACIENTES Y MÉTODOS}

Investigación descriptiva, transversal y correlacional. La población del estudio estuvo constituida por 49 parejas formadas por un hombre entre 40 y 65 años y su pareja una mujer entre 40 y 60 años usuaria de un centro de salud de la ciudad de Concepción, Chile. Se consideraron las edades de 40-60 años en la mujer y de 40-65 años en los hombres en base a la revisión de la literatura. El procedimiento de selección de la muestra se describió en un artículo previo relativo a la percepción de las mujeres y sus parejas sobre la calidad de vida en el climaterio (11). Los criterios de inclusión fueron: parejas formadas por un hombre entre $40 \mathrm{y}$ 65 años y una mujer entre 40-60 años; presentar un estado de salud compatible con el desempeño de las actividades rutinarias; encontrarse sin licencia médica al momento de la aplicación del cuestionario y haber otorgado su consentimiento informado para participar en la investigación.

Para la evaluación de la calidad de vida se utilizó la Escala Aging Males' Symptoms (AMS) (5). La alteración en la calidad de vida fue clasificada de acuerdo a la puntuación obtenida en la escala total y en las distintas sub-escalas de acuerdo a lo publicado por Heinemann y cols (5). En la escala global se clasificó como ninguna alteración en la calidad de vida puntajes $<26$, como leve a puntajes entre 27 a 36, moderada 37 a 49 y severa 50 o más. En la sub-escala psicológica se clasificó como ninguna alteración $<5$, leve 6-8, moderada 9 a 11 y severa 12 o más. En la sub-escala somatovegetativa fue clasificada como ninguna alteración puntajes $<8$, leve 9-12, moderada 13 a 18, y severa 19 o más. Finalmente la puntuación en la sub-escala sexual se clasificó como ninguna alteración puntajes $<5$, leve 6-7, moderada 8 a 10 y severa 11 o más.

La escala fue aplicada a cada uno de los miembros de la pareja, para de este modo conocer la percepción del hombre respecto de su calidad de vida durante esta etapa y además la percepción que la pareja tiene respecto de la calidad de vida de él. Los instrumentos fueron administrados simultáneamente en privado, solicitándoles no realizar comentarios ni preguntas a su pareja mientras duraba la aplicación de los mismos.

El proyecto fue sometido a la consideración del Comité de Ética de la Facultad de Medicina de la Universidad de Concepción. Posteriormente 
se solicitó autorización a las autoridades de la Dirección de Salud de Concepción (DAS), así como al Director del Centro de Salud donde se realizó el trabajo.

La recolección de datos se realizó en el domicilio de la pareja por las autoras, con la colaboración de dos encuestadores previamente entrenados. Al momento de la aplicación de los instrumentos ambos miembros de la pareja debían encontrarse en el domicilio y otorgar su consentimiento informado.

Para el análisis estadístico los datos fueron procesados utilizando el programa estadístico SPSS 12.0. Se utilizó estadística descriptiva, índice de Kappa y t de Student. Se consideró como diferencia significativa valores de $p<0,05$.

\section{RESULTADOS}

La Tabla I muestra las características biodemográficas de los(as) participantes del estudio. La totalidad de los(as) participantes refirieron estar casados. El $32,7 \%$ de los hombres y $28,6 \%$ de las mujeres señalaron tener escolaridad media completa y la mayor parte de los participantes refirió ser beneficiario del Fondo Nacional de Salud (FONASA). Casi la mitad de los hombres y la mayoría de las mujeres consumen algún tipo de medicamentos.

La Tabla II muestra la distribución en porcentaje de los hombres según la severidad de los síntomas en las sub-escalas y en la escala global. De acuer- do a los puntos de corte explicitados previamente, el $54,8 \%$ de los hombres mostró alguna alteración de la calidad de vida, correspondiendo un $35,7 \%$ a leve, $9,5 \%$ moderada y $9,5 \%$ severa, mostrando mayor alteración en las sub-escalas somatovegetativa y sexual.

En promedio, los síntomas percibidos como más intensos en las distintas sub-escalas fueron: irritabilidad (sub-escala psicológica), dolor articulaciones y dolor muscular, agotamiento físico, y disminución de la fuerza muscular (sub-escala somatovegetativa) y disminución del rendimiento sexual y disminución de erecciones matinales (sub-escala sexual).

Mediante el índice de Kappa (12) se analizó la concordancia entre la percepción de los hombres y la percepción de sus parejas sobre la presencia de síntomas en las distintas sub-escalas (Tabla III). Luego se interpretó de acuerdo a la clasificación propuesta por Landis y Koch (12), considerando valores de Kappa inferiores a 0 como grado de acuerdo pobre, entre 0 y 0,20 leve, 0,21 y 0,40 discreto, entre 0,41 y 0,60 moderado, entre 0,61 y 0,80 sustancial y entre 0,81 y 1 casi perfecto.

Para los síntomas de la sub-escala psicológica se encontró una concordancia altamente significativa y un grado de acuerdo sustancial para estado de ánimo depresivo, altamente significativa y moderada para se siente hundido y significativa y discreta para irritabilidad y ansiedad.

Tabla I

CARACTERÍSTICAS DEMOGRÁFICAS DE LOS (AS) PARTICIPANTES DEL ESTUDIO

\begin{tabular}{|c|c|c|c|c|c|}
\hline \multirow{3}{*}{$\begin{array}{l}\text { Variable } \\
\text { Edad (promedio) }\end{array}$} & & \multirow{2}{*}{\multicolumn{2}{|c|}{$\begin{array}{l}\text { Hombres } \\
53,2 \pm 8,1\end{array}$}} & \multirow{2}{*}{\multicolumn{2}{|c|}{$\begin{array}{c}\text { Parejas } \\
\text { (Mujeres) } \\
50,8 \pm 6,9\end{array}$}} \\
\hline & & & & & \\
\hline & & $\mathrm{n}$ & $\%$ & $\mathrm{n}$ & $\%$ \\
\hline \multirow{7}{*}{$\begin{array}{l}\text { Casados } \\
\text { Escolaridad }\end{array}$} & & 49 & 100 & 49 & 100 \\
\hline & Sin escolaridad & 1 & 2 & 0 & 0 \\
\hline & Básica incompleta & 8 & 16,3 & 9 & 18,4 \\
\hline & Básica & 16 & 32,7 & 21 & 42,8 \\
\hline & Media & 16 & 32,7 & 14 & 28,6 \\
\hline & Técnico-Profesional & 5 & 10,2 & 5 & 10,2 \\
\hline & Universitaria & 3 & 6,1 & 0 & 0 \\
\hline \multirow[t]{4}{*}{ Previsión de salud } & FONASA & 40 & 81,6 & 38 & 77,6 \\
\hline & ISAPRE & 2 & 4,1 & 2 & 4,1 \\
\hline & Sin previsión & 5 & 10,2 & 7 & 14,3 \\
\hline & Otro & 1 & 2,0 & 1 & 2,0 \\
\hline \multicolumn{2}{|c|}{ Uso de medicamentos } & 23 & 46,9 & 35 & 71,4 \\
\hline
\end{tabular}

FONASA: Fondo Nacional de Salud. ISAPRE: Institución de Salud Previsional 
Tabla II

DISTRIBUCIÓN DE LA ALTERACIÓN DE LA CALIDAD DE VIDA EN HOMBRES PARTICIPANTES DEL ESTUDIO

\begin{tabular}{lccc}
\hline & & \multicolumn{2}{c}{ Alteración de la calidad de vida } \\
& Ninguna & Pequeña & Severa \\
\hline Escala total $(n=42)$ & 45,2 & 35,7 & 9,5 \\
Psicológica $(n=47)$ & 10,6 & 63,8 & 8,5 \\
Somatovegetativo(n=49) & 4,1 & 38,8 & 34,7 \\
Sexual $(n=44)$ & 18,2 & 15,9 & 36,4 \\
\hline
\end{tabular}

Tabla III

CONCORDANCIA ENTRE SÍNTOMAS PERCIBIDOS POR LOS HOMBRES Y POR SUS PAREJAS

\begin{tabular}{llcc}
\hline Sub-escala & Síntomas & Kappa & Valor p \\
\hline Psicológica & Irritabilidad & 0,297 & ${ }^{*} 0,038$ \\
& Nerviosismo & 0,062 & 0,648 \\
& Ansiedad & 0,326 & ${ }^{*} 0,024$ \\
& Estado de ánimo depresivo & 0,620 & ${ }^{* *} 0,000$ \\
& Se siente hundido & 0,516 & ${ }^{* *} 0,000$ \\
Someto-vegetativa & Disminución sensación bienestar general & 0,366 & ${ }^{*} 0,010$ \\
& Dolor articulaciones y dolor muscular & 0,496 & ${ }^{*} 0,000$ \\
& Sudor excesivo & 0,341 & ${ }^{* *} 0,002$ \\
& Problemas de sueño & 0,447 & 0,101 \\
Sexual & Mayor necesidad dormir/cansado & 0,233 & 0,804 \\
& Agotamiento físico/falta vitalidad & $-0,031$ & 0,312 \\
& Disminución de la fuerza muscular & 0,124 & ${ }^{*} 0,025$ \\
& Sensación que ha pasado mejor momento de su vida & 0,318 & 0,131 \\
& Disminución crecimiento de la barba & 0,200 & ${ }^{* *} 0,001$ \\
& Disminución capacidad/frecuencia rendimiento sexual & 0,514 & ${ }^{* *} 0,005$ \\
& Disminución número erecciones matinales & 0,433 & ${ }^{* *} 0,000$ \\
\hline
\end{tabular}

${ }^{*} p<0,05 .{ }^{* *} p<0,01$.

Para los síntomas de la subescala somatovegetativa se encontró una concordancia altamente significativa y un grado de acuerdo moderado para dolor articulaciones y dolor muscular y problemas de sueño, y una concordancia significativa y un grado de acuerdo discreto para disminución sensación bienestar general y sudor excesivo.

Para los síntomas de la sub-escala sexual se encontró una concordancia altamente significativa entre ambas percepciones, siendo el grado de acuerdo moderado para disminución capacidad/ frecuencia rendimiento sexual, disminución número erecciones matinales, disminución del deseo sexual, y significativa con un grado de acuerdo discreto para sensación que ha pasado el mejor momento de su vida. En donde del total de hombres que no perciben que ha pasado el mejor momento de su vida, casi dos tercios coincide con la percepción de sus parejas y del total de hombres que si perciben que ha pasado su mejor momento, el $60 \%$ coincide con la percepción de sus parejas.

Al comparar los puntajes de los hombres en las tres sub-escalas con la percepción de sus parejas, sólo se encontró diferencia significativa en la subescala psicológica $(p<0,01)$, mientras que en las sub-escalas somatovegetativa, sexual y en la escala total no se encontraron diferencias significativas (Tabla IV). La Figura 1 ilustra los puntajes promedio de las tres sub-escalas de la escala AMS en los hombres y la percepción que sus parejas tienen de la intensidad de los síntomas de ellos. 
Tabla IV

COMPARACIÓN ENTRE PUNTAJES POR SUBESCALAS DE LA CALIDAD DE VIDA EN EL CLIMATERIO MASCULINO EN HOMBRES Y PERCEPCIÓN DE SUS PAREJAS

\begin{tabular}{|c|c|c|c|c|c|c|}
\hline Sub-escala & & $\mathrm{n}$ & promedio & $\pm \mathrm{DE}$ & $\mathrm{t}$ & Valor $p$ \\
\hline Psicológica & $\begin{array}{l}\text { Hombre } \\
\text { Pareja }\end{array}$ & $\begin{array}{l}46 \\
46\end{array}$ & $\begin{array}{c}8,6957 \\
10,1957\end{array}$ & $\begin{array}{l}3,84054 \\
4,68861\end{array}$ & $-3,07$ & ${ }^{* *} 0,004$ \\
\hline Somatovegetativa & $\begin{array}{l}\text { Hombre } \\
\text { Pareja }\end{array}$ & $\begin{array}{l}48 \\
48\end{array}$ & $\begin{array}{l}14,8333 \\
14,9583\end{array}$ & $\begin{array}{l}5,47075 \\
6,07036\end{array}$ & $-0,17$ & 0,869 \\
\hline Sexual & $\begin{array}{l}\text { Hombre } \\
\text { Pareja }\end{array}$ & $\begin{array}{l}40 \\
40\end{array}$ & $\begin{array}{l}9,2250 \\
9,5500\end{array}$ & $\begin{array}{l}4,11680 \\
4,21201\end{array}$ & $-1,01$ & 0,318 \\
\hline Total & $\begin{array}{l}\text { Hombre } \\
\text { Pareja }\end{array}$ & $\begin{array}{l}36 \\
36\end{array}$ & $\begin{array}{l}32,1944 \\
33,5833\end{array}$ & $\begin{array}{l}12,72303 \\
13,47882\end{array}$ & $-1,12$ & 0,272 \\
\hline
\end{tabular}

${ }^{*} \mathrm{p}<0,05 .{ }^{* *} \mathrm{p}<0,01$.

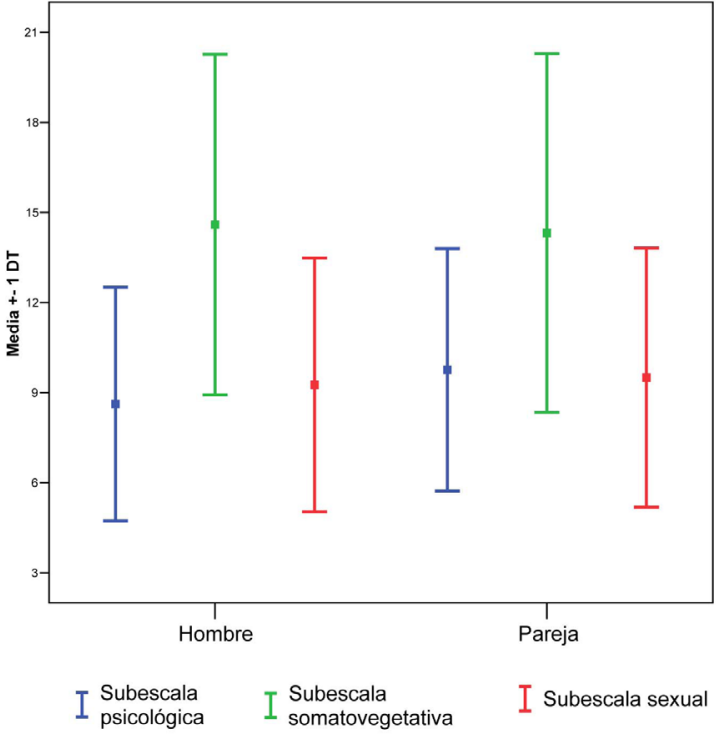

Figura 1. Comparación entre puntajes por sub-escalas de la calidad de vida en el climaterio masculino en hombres y percepción de sus parejas.

\section{DISCUSIÓN}

En este estudio se encontró que en las sub-escalas somatovegetativa y sexual, más de la mitad de los hombres presentó un deterioro moderado y severo de su calidad de vida, siendo este porcentaje menor en la sub-escala psicológica. En general, la comparación con otros estudios que utilizaron la escala AMS, muestra un mayor deterioro en los hombres chilenos que lo reportado en Europa $(5,6,8)$ y similares resultados, sólo en la subes- cala sexual, que lo encontrado en Japón (7), en donde más de la mitad de los hombres japoneses presentaron una alteración moderada o severa en esta sub-escala. Respecto de un estudio realizado en Brasil (10), la calidad de vida reportada por los hombres en ese estudio fue similar es la situación descrita en este trabajo.

Al examinar la concordancia encontrada entre la percepción de los hombres y sus parejas sobre la presencia de síntomas, distintos resultados se obtuvieron en cada sub-escala. En la psicológica sólo se encontró un alto grado de acuerdo para estado de ánimo depresivo y se siente hundido. En adición a ello se encontraron diferencias significativas en la percepción de intensidad de los síntomas en esta sub-escala entre ambos miembros de la pareja. Las diferencias encontradas pueden hacer presumir la falta de comunicación en la pareja respecto de la vivencia de síntomas. Ello es consistente con lo señalado por autoras colombianas (13) en un estudio de abordaje cualitativo en donde entrevistaron a un grupo de académicos entre 50 y 60 años, los que señalaron que respecto de la andropausia: "no hablamos usualmente del tema", "yo no me he dado cuenta que ella haya percibido mis cambios", "nunca toco el tema con mi compañera", "tema de no tocar". Los hallazgos señalan que aunque los hombres no comunican su situación están pendientes de captar cualquier información que les permita fortalecer la impresión positiva de los cambios surgidos.

El grado de acuerdo moderado encontrado en la sub-escala somatovegetativa para dolor articulaciones y dolor muscular y problemas de sueño resulta razonable dado que el dolor provoca, en quién lo experimenta, quejas y malestar, disminuye la movilidad e impide el desarrollo de actividades habituales, por lo que estas serían manifestaciones evi- 
dentes y observables por la pareja. Respecto de los problemas de sueño, éstos también son fácilmente perceptibles por las parejas, ya que esta reportado que los problemas en este ámbito no sólo afectan al que los padece, sino también a su acompañante, cuyo sueño es perturbado (14).

Respecto de los síntomas del dominio sexual se encontró un grado de acuerdo moderado para disminución de la capacidad/frecuencia de rendimiento sexual, disminución del número de erecciones matinales y disminución del deseo sexual. El grado de acuerdo encontrado entre las percepciones de ambos miembros de la pareja en esta sub-escala, coincide con los resultados obtenidos al comparar la percepción de los hombres respecto del impacto de los síntomas con la percepción de sus parejas. Especial atención requiere este hallazgo, ya que ambos miembros de la pareja coinciden, en alguna medida, en el grado de acuerdo referente a la presencia de los síntomas y en el impacto que tienen en la calidad de vida relacionada con salud del hombre, lo que da cuenta que en esta sub-escala las mujeres están percibiendo lo que le ocurre a sus parejas. Esto obviamente se debe a que este ámbito es el de mayor intimidad y donde los síntomas, como la disminución de la capacidad de rendimiento sexual, del deseo y erección, que afectan al hombre también causan un impacto directo en su pareja.

Lo anterior podría reflejar que para la mayoría de los hombres y las mujeres, la sexualidad es un aspecto muy valorado de su calidad de vida, y un menoscabo en este ámbito puede causar desarmonía en la pareja $(11,15)$. En este punto cabe mencionar que existen estudios que señalan que la disfunción sexual puede originar conflictos y daños en la relación de la pareja (16).

\section{CONCLUSIÓN}

Existe un porcentaje importante de hombres que presentan alteración en su calidad de vida. Es llamativo que las percepciones de hombres y sus parejas relativas a la condición de climaterio masculino sean similares en cuanto a los aspectos somatovegetativo y sexual, y diferentes en el aspecto psicológico. Es necesario realizar nuevas investigaciones que permitan determinar si una muestra mayor se comporta de manera similar. El estudio de los aspectos señalados es particularmente importante ya que permitirá, por una parte, determinar el impacto que la sintomatología de este período del ciclo vital tiene en la calidad de vida de los hombres, y de este modo el desarrollar estrategias para que la población masculina aprenda a aceptar el envejecimiento de una mejor manera y a buscar ayuda o soluciones, si es necesario, promoviendo un mayor período de vida activa y saludable (17). Finalmente, el conocimiento que los hombres y sus parejas tengan de los factores que inciden en este proceso y su enfrentamiento, contribuiría a suprimir estereotipos, fortalecer la relación de pareja y el autocuidado mutuo con repercusiones importantes en la salud de la pareja.

\section{REFERENCIAS}

1. Devoto E, Aravena L. Hipogonadismo asociado a la senilidad en el varón (climaterio masculino - andropausia - ADAM). Rev Chil Obstet Ginecol 2004;69:392-8.

2. Werner AA. Male climateric: report of two hundred and seventy-three cases. J Am Med Assoc 1946;132:18894.

3. Castelo Elias-Calles L, Machado Porro MC. Algunas consideraciones sobre el síndrome de PADAM. Rev Cubana Endocrinol 2006;17:24-36.

4. Morales A, Heaton J, Carson C 3rd. Andropause: a misnomer for a true clinical identity. J Urol 2000;163:705-12.

5. Heinemann LAJ, Zimmermann T, Vermeulent A, Thiel C, Hummel W. A new "aging males' symptoms" rating scale. Aging Male 1999;2:105-14.

6. Myon E, Martin N, Taïeb CH. The French Aging Males' Symptoms (AMS) scale: Methodological review. Health Qual Life Outcomes 2005;3:20.

7. Ichioka K, Nishiyama H, Yoshimura K, Itoh N, Okubo $\mathrm{K}$, Terai A. Aging males' symptoms scale in Japanese men attending a multiphasic health screening clinic. Urology 2006;67:589-93.

8. Valenti G, Capone M, Forti G, Grasso M, Mirone V, Chiaffarino $\mathrm{F}$, et al. Inverse relationship between scores on the quality of life questionnaire SF-12 and on the Aging Males' Symptoms scale in Italian men. Aging Male 2008;11:77-82.

9. Abdo CHN, Afif-Abdo J. Estudo populacional do envelhecimento (EPE): primeiros resultados masculinos. Rev Bras Med 2007;64:379-83.

10. Corrêa LQ, Rombaldi AJ, Da Silva MC, Domingues MR. Aging male's symptoms in a Southern Brazil population: lifestyle effects after the age of 40 . Aging Male 2010;13:93-9.

11. Salazar A, Paravic T. Barriga O. Percepción de las mujeres y sus parejas sobre la calidad de vida en el climaterio. Rev Chil Obstet Ginecol 2011;76:64-70.

12. Landis JR, Koch GG. The measurement of observer agreement for categorical data. Biometrics 1977;33:159-74.

13. Rosalez E, Rojas P. Andropausia y sexualidad. ¿Una paradoja? Colombia Médica 2000;31:16-9.

14. National Sleep Foundation. 2005 Sleep in America Poll. Washington: National Sleep Foundation; 2005. Hallado en: http://www.sleepfoundation.org/sites/default/files/2005_summary_of_findings.pdf. Acceso en junio 2011.

15. Hodson P. Male sexual function and its problems. En: 
Tomlinson JM (ed). Sexual Health and Menopause. London: Royal Society Medicine Press Ltd.; 2005. pp 63.

16. Blümel JE, Castelo-Branco C, Cancelo MJ, Romero H, Aprikian D, Sarra S. Impairment of sexual activity in middle-aged women in Chile. Menopause 2004;11:7881.

17. Gooren LJG. Quality-of-life issues in the aging male. Aging Male 2000;3:185-9. 